\title{
Gentrificación contemporánea y derecho a la ciudad: la defensa del espacio urbano en la Ciudad de México
}

Contemporary gentrification and the right to the city: The defense of the urban space in Ciudad de México

\section{Gabriel Gómez Carmona*}

Recibido: 28 de marzo de 2018

Aceptado: 14 de diciembre de 2018

\section{Resumen}

En las últimas décadas las ciudades a nivel mundial han sido objeto de profundas transformaciones producto de las políticas públicas y la normatividad aplicadas a su desarrollo, al modelo dominante de urbanización capitalista y los complejos cambios socioespaciales que experimenta. Estas situaciones generan tensiones entre los diferentes actores que habitan la ciudad, expresándose en movimientos ciudadanos de defensa del espacio urbano, el entorno y los recursos naturales del territorio. A través de una etnografía digital, el artículo analiza cómo la defensa del espacio urbano ante el embate de la gentrificación contemporánea constituye una de las principales luchas de los movimientos ciudadanos de la Ciudad de México para hacer valer su Derecho a la Ciudad. Como resultados, se establece que este derecho al estar consagrado en la Constitución Política de la Ciudad de México como un derecho humano fundamental más, marca el inicio de una nueva etapa donde la participación ciudadana podrá incidir en el rumbo de las políticas públicas aplicadas al futuro desarrollo urbano de la ciudad.

Palabras clave: apropiación del espacio, derecho a la ciudad, etnografía digital, gentrificación, identidad urbana.

\begin{abstract}
In recent decades, cities worldwide have undergone profound transformations as a result of public policies and regulations applied to their development, to the dominant model of capitalist urbanization and the complex socio-spatial changes that it experiences. These situations generate tensions between the different actors that inhabit the city, expressing themselves in citizen movements defending the urban space, the environment and the natural resources of the territory. Through a digital ethnography, the article analyzes how the defense of the urban space before the onslaught of contemporary gentrification, constitutes one of the main struggles of the citizen movements of Mexico City to assert its Right to the City. As results, it is established that this right to be enshrined in the Political Constitution of Mexico City as a fundamental human right, marks the beginning of a new stage where citizen participation may influence the direction of public policies applied to the future urban development of the city.
\end{abstract}

Keywords: appropriation of space, digital ethnography, gentrification, right to the City, urban identity.

* Filiación: Universidad La Salle, Ciudad de México, México. Contacto: gabriel.gomez@lasalle.mx

El presente artículo es parte del proyecto de investigación “El Derecho a la Ciudad y la gentrificación de espacios centrales en la CDMX en el contexto de la reconstrucción post-catástrofe 19S2017" (IMC-09/18) que actualmente se desarrolla en la Facultad Mexicana de Arquitectura, Diseño y Comunicación (FAMADYC) de Universidad La Salle, México, con fondos institucionales.

Cómo citar: Gómez-Carmona, G. (2018). Gentrificación contemporánea y derecho a la ciudad: la defensa del espacio urbano en la Ciudad de México. Revista de Urbanismo, 39, 1-14. https://doi.org/10.5354/0717-5051.2018.48816 


\section{Introducción}

Actualmente las ciudades en toda su complejidad se constituyen como una de las obras culturales más distintivas de la historia de la civilización, pues además de representar la memoria viva de una sociedad, en su evolución sintetizan su desarrollo social, político, económico, tecnológico y cultural a través del tiempo (Ascher, 2004).

El espacio urbano puede entenderse como todo aquello creado con un fundamento y un sentido social, más allá de un conjunto de edificaciones erigidas de manera técnica, institucionalizada e instrumental (Aguilar, 2012). Esto lo lleva a una categoría superior al incorporarlo al universo simbólico de cualquier grupo humano, es decir, como parte del enorme conjunto de significaciones, simbolismos e imaginarios en los que la colectividad se encuentra e identifica en su cotidianidad, con experiencias propias y comunes que dan sentido a la vida de sus ciudadanos, es ahí donde reside la trascendencia de la ciudad como fenómeno cultural.

A pesar de ello, en las últimas décadas a nivel global, las ciudades fueron objeto de múltiples fenómenos que transformaron de manera irreversible el espacio urbano, al grado de conferirles una nueva dimensión socioespacial, lo que reconfiguró no sólo su territorio, sino la manera en la que las vivimos, significamos y apropiamos, dando como resultado, nuevos y complejos conglomerados que caracterizan a escala planetaria la realidad urbana actual.

El presente artículo aborda cómo la defensa del espacio urbano ante el embate de la gentrificación constituye una de las principales luchas de los movimientos ciudadanos de la Ciudad de México (CDMX) para hacer valer su Derecho a la Ciudad, definido por la Constitución Política de la Ciudad de México (2017) en su artículo 12, como:

[E]l derecho colectivo que garantiza el ejercicio pleno de los derechos humanos, la función social de la ciudad, su gestión democrática y asegura la justicia territorial, la inclusión social y la distribución equitativa de bienes públicos con la participación de la ciudadanía (p. 46).

Lo anterior se ejemplifica con el seguimiento de la cobertura periodística realizada a diversos movimientos ciudadanos generados en la CDMX durante el 2018, lo que deja comprender a la luz de la teoría y de la perspectiva cualitativa de la etnografía digital, la importancia de dichos temas al interior de la Antropología Urbana, al generar descripciones detalladas de la forma en que se transforma el entorno urbano como consecuencia de las dinámicas sociales, políticas, económicas y culturales, que tienen lugar en la ciudad, así como las repercusiones de dichos cambios.

\section{Ciudad, Identidad y la apropiación simbólica del espacio}

Visualizar la ciudad como objeto de estudio de las Ciencias Sociales permite reconocerla como parte esencial del proceso sociocultural de todo grupo humano, lo que habla de una sociedad espacializada portadora de un enorme conjunto de significados $y$ significantes que van desde la ciudadanía, el espacio urbano y el habitar (Giglia, 2012), hasta el consumo, la exclusión y la identidad (Magrini y Cataláo, 2017) por mencionar algunos. Estos representan puntos de partida para el estudio de múltiples temas relacionados con la ciudad, su crecimiento y la forma en la que es habitada, simbolizada y apropiada por parte de sus habitantes. Es el espacio y el tiempo que vivimos lo que nos define, es donde nos conformamos como seres, donde constituimos nuestra identidad y construimos nuestra personalidad y particular visión del mundo (Tamayo y Wildner, 2005, p. 20), es lo que permite generar ese vínculo fundamental entre espacio urbano e individuos.

A pesar de que la identidad ha sido un tema de reflexión y debate a través de la historia, ésta no puede encasillarse de manera rígida en un solo concepto, por ser un fenómeno en continua construcción, de ahí que sea mejor referir algunas de sus principales características, como el hecho de que la identidad puede verse como un proceso dinámico y flexible, que debe estudiarse a través del tiempo (Alva y Aldrete, 2011) y que se emplea como un sentimiento de pertenencia, que se centra en la relación dialéctica entre el individuo y la sociedad (Waldmann, 2011).

De esta manera la cultura como resultado del proceso de organización social sobre el espacio (Alva y Aldrete, 2011) influye de manera directa en la configuración del territorio y en el diseño de los espacios urbanoarquitectónicos que habitamos y socializamos, lo que 
transforma irreversiblemente el entorno por el hecho de que los individuos y la colectividad al dejar su huella material sobre el espacio, le confieren un conjunto de señales y marcas cargadas de simbolismos (Vidal y Pol, 2005, p. 283) que finalmente, dotan al espacio de identidad, lo que deja ver la estrecha relación existente entre la ciudad, el espacio urbano y la construcción de identidad.

La relación que los individuos establecen con los espacios que habitan, permite la creación de identidades urbanas, derivadas a su vez, del sentimiento de pertenencia o afiliación a un entorno significativo, resultando una categoría social más de las diversas que empleamos para definir nuestra identidad social (Valera, 1996, p. 6). Es en esa relación cercana con nuestro entorno, que la identidad se materializa en los espacios que se viven en la cotidianidad, es decir, en el día a día con el resto de los ciudadanos, vecinos, conocidos y amigos con los que entablamos relaciones cercanas de afecto o bien, funcionales (producción, intercambio, consumo) en esos espacios de la ciudad.

Únicamente cuando un grupo humano se siente identificado con el entorno, su historia y los espacios que vive puede desarrollar fuertes vínculos con estos elementos que, a su vez, se manifiestan en lazos de unión entre los individuos del propio grupo, lo que en conjunto constituye su identidad urbana. Cuando el espacio urbano rebasa la mera categoría del soporte físico de las actividades humanas en el territorio y crea procesos de identificación, cercanía y complementariedad con los individuos que lo habitan se produce una apropiación simbólica de estos espacios pues, "permiten a los individuos y grupos establecer una interacción dinámica con el entorno, apropiarse de él y establecer un sentimiento de pertenencia" (Valera, 1996, p. 16).

El proceso de apropiación e identificación simbólica con el espacio no es un proceso rígido, ni cerrado, por el contrario, es un proceso elástico y abierto, siempre en continua transformación, razón por la cual, la identidad urbana implica también la continua construcción social del espacio urbano, a la par de las dinámicas sociales, políticas, económicas y culturales que transforman el entorno, lo que invariablemente genera fuertes tensiones entre los diferentes actores sociales que habitan la ciudad, situación que puede desembocar en múltiples conflictos con un punto en común: la disputa y control del espacio urbano, por el hecho de que son los individuos y las colectividades quienes lo habitan, socializan, simbolizan y apropian.

\section{Gentrificación contemporánea, Derecho a la Ciudad y la defensa del espacio urbano}

En las últimas décadas diversas zonas centrales de grandes ciudades a nivel global fueron objeto de intervenciones que tendieron a la redensificación de estas, buscando la mejora de su espacio urbano y el incremento de su stock de vivienda. Entre los aspectos que influyen decisivamente en la realización de estas acciones se encuentran: los privilegios de localización, el equipamiento y redes de infraestructura consolidadas, además de su condición histórica y patrimonial.

Aunque esto significa un cambio en la imagen y la estructura urbana de las ciudades, también representa fuertes impactos en aspectos económicos, culturales, pero ante todo, sociales, pues la evidencia empírica muestra (Contreras, 2017; Gómez-Carmona y VillarCalvo, 2015; Gómez-Carmona, Villar-Calvo e InzulzaContardo, 2016; Inzulza-Contardo y Díaz, 2016; InzulzaContardo y Galleguillos, 2014; Janoschka, Sequera \& Salinas, 2013) que no siempre este conjunto de transformaciones urbanas implican la mejora de las condiciones de vida de todos los sectores de la población, al grado de que en diversas ciudades, se pueden constatar fenómenos de gentrificación.

Los estudios coinciden en que la gentrificación implica un fuerte impacto social, por el hecho de que es un concepto que define procesos de elitización de zonas de la ciudad que son rehabilitadas para atraer nuevos usuarios y habitantes de estratos socioeconómicos más altos a los de la población que originalmente las habita, porque su nivel adquisitivo les permite pagar mayores precios por el suelo, la vivienda, el comercio y los nuevos servicios instalados.

La consecuencia es que los habitantes "oriundos" de estas zonas (antes populares), son expulsados o ven mermada su calidad de vida, lo que nos cuestiona si ¿Es posible la mejora y redensificación de espacios centrales de la ciudad sin gentrificarlos? 
La ciudad que hoy día venden los medios de comunicación y la mercadotecnia inmobiliaria engrandece el espacio privado, donde la plaza comercial o los exclusivos mega desarrollos inmobiliarios mixtos, producen una ciudad gentrificada y fragmentada, en la que estos objetos urbano-arquitectónicos sustituyen a las plazas públicas como los grandes concentradores de actividades y encuentro en la ciudad, convirtiendo la movilidad en un desplazamiento fugaz entre el ocio y el consumo (Montaner y Muxí, 2011). De tal suerte, que el espacio público se ve alterado por nuevos usos que el capital inmobiliario le asigna de acuerdo a su potencial económico; de ser un lugar de encuentro y socialización de la población, poco a poco se convierte en espacio privado de consumo, accesible solo a aquellos que pueden pagar la nueva oferta de vivienda, servicios y comercio instalado, encareciendo el suelo para amplios sectores de la población que la mayoría de las veces, se ven obligados a salir de estas zonas revaloradas y elitizadas.

Esto da cuenta de un novedoso derecho a la ciudad en el cual, aquellos que tienen un mayor poder adquisitivo tienen acceso a un consumo de bienes $y$ servicios terciarios selecto, lo que se traduce en un proceso de empoderamiento del ciudadano en la construcción de su identidad, frente a aquellos que son excluidos e invisibilizados de estos nuevos espacios de consumo por no tener los recursos económicos necesarios para acceder a ellos, lo que en términos generales, plantea la necesidad de mirar el consumo como condición necesaria para entender al individuociudadano, como alguien que tiene derecho a tener derechos en la ciudad actual (Magrini, y Cataláo, 2017).

De esta forma, el Derecho a la Ciudad definido como:

[u]n derecho colectivo de los habitantes de las ciudades, en especial de los grupos vulnerables y desfavorecidos, que les confiere legitimidad de acción y de organización, basado en sus usos y costumbres, con el objetivo de alcanzar el pleno ejercicio del derecho a la libre autodeterminación y un nivel de vida adecuado (Carta Mundial por el Derecho a la Ciudad, 2013, p. 369)

se ve banalizado como una mercancía que se puede comprar, sujeto a la oferta y la demanda, lo que invisibiliza a inmensos sectores de la población que ven vulnerados sus derechos humanos fundamentales por este proceso.

A pesar del cuestionamiento en diversos ámbitos (legal, antropológico, sociológico) sobre la práctica y cumplimiento del Derecho a la Ciudad, en los últimos años la discusión ha ganado varios espacios hasta constituirse en una verdadera demanda social su respeto y efectivo cumplimiento, al grado de que representa uno de los grandes desafíos urbanos planetarios hacia el primer cuarto del siglo XXI por la sencilla razón de que la vivienda, el empleo, la movilidad, el acceso a áreas verdes, servicios y espacios públicos de calidad entre otros, son necesidades urbanas que deben satisfacerse y garantizarse como parte fundamental de ese Derecho a la Ciudad para todos los habitantes y consagrado en la Declaración de Quito sobre Ciudades y Asentamientos Humanos Sostenibles para Todos, mejor conocida como Nueva Agenda Urbana (2017).

La Nueva Agenda Urbana conjunta las principales necesidades urbanas a atender en cualquier ciudad, como parte del compromiso de todos los organismos, agencias y gobiernos firmantes de dicha declaración en la cumbre Hábitat III, celebrada en Quito, Ecuador, en 2016.

Sin embargo, para el caso de los países pobres o periféricos, la mayoría de estas necesidades representan fuertes deudas históricas con su población, al grado de que no se les visualiza una clara, ni pronta solución en el mediano o largo plazo, de ahí que múltiples movimientos ciudadanos resurgen en diversas ciudades del planeta como voces críticas del modelo urbano capitalista para exigir el respeto y cumplimiento para todos los habitantes, en especial de los más desfavorecidos, del Derecho a la Ciudad a través de la defensa del espacio urbano y del territorio de sus barrios, comunidades y colonias ante el agresivo avance del desarrollo inmobiliario que trastoca las características y usos tradicionales de su espacio urbano al gentrificarlo.

\section{Metodología}

El trabajo se desarrolla desde un enfoque cualitativo, utilizando las técnicas ciberantropológicas o de la etnografía digital (Flores-Vivar, 2018) que permiten no sólo acercarse sino, documentar la realidad social a través del flujo de información en internet y los medios 
digitales. Este nuevo abordaje metodológico desde las Ciencias Sociales facilita una nueva lectura de la realidad, por el hecho de permitir a la sociedad misma formar parte activa del proceso de construcción de la información y al investigador, disponer a través de los medios digitales, de ese enorme banco de información (Flores-Vivar, 2018). De esta manera, el proceso de investigación se convierte en una experiencia hipertextual, que engloba la complejidad del estar en la red, ya sea navegando, consultando datos, usando buscadores, leyendo o recuperando información (Corona-Rodríguez, 2013) lo que constituye una nueva experiencia de análisis e interpretación de la enmarañada realidad de las ciudades contemporáneas y sus múltiples actores.

Para ello, se analiza la cobertura que el periódico de circulación nacional La Jornada, realizó en sus secciones Capital y Sociedad entre el 14 de febrero y el 05 de diciembre de 2018, a diversos movimientos ciudadanos que tuvieron lugar en la $\operatorname{CDMX}$ y que de manera específica luchan por la defensa del territorio y los recursos (suelo y agua) de sus barrios, comunidades y colonias ante el fuerte avance del desarrollo inmobiliario que ha modificado de manera irreversible el espacio urbano en los últimos seis años. Se analizan también notas que documentan las acciones que la nueva legislatura y la administración local realizan para dar solución a las demandas ciudadanas ante dicha problemática. Todo el seguimiento se realizó a través de la página electrónica del periódico La Jornada, lo que permitió el análisis diacrónico de la cobertura informativa, a su vez, ésta se contrasta con datos oficiales del Gobierno local, dependencias federales y recorridos de las zonas estudiadas.

El andamiaje teórico construido para este artículo se basa en los conceptos de identidad urbana, apropiación simbólica del espacio, gentrificación y Derecho a la Ciudad, aplicados a eventos y lugares específicos, por lo que no se busca generalizar los casos expuestos, por el contrario, se busca analizar ejemplos que muestren la forma en la que procesos comunes a diversas realidades planetarias se gestan de manera particular en una ciudad latinoamericana, en este caso, la CDMX. El artículo constituye la primera parte del proyecto de investigación El Derecho a la Ciudad y la gentrificación de espacios centrales en la CDMX en el contexto de la reconstrucción post-catástrofe $19 S 2017$ (IMC-09/18) que actualmente se desarrolla en la Facultad Mexicana de Arquitectura, Diseño y Comunicación (FAMADYC) de Universidad La Salle, México, por lo que los resultados presentados, son parciales.

\section{La defensa del espacio urbano: el caso de la CDMX}

Para ejemplificar este recorrido teórico presentaremos como caso de estudio, las tensiones generadas entre diversos grupos ciudadanos, organizaciones vecinales, gobierno local y empresas inmobiliarias durante el 2018 en varias alcaldías de la Ciudad de México (CDMX), por la autorización, desarrollo y construcción de exclusivos complejos inmobiliarios (vivienda, comercio, servicios) que vienen a transformar de manera agresiva no sólo el entorno urbano, sino la conformación social, económica y cultural de las barrios, comunidades, colonias y pueblos tradicionales donde se establecen.

Para iniciar, precisaremos que la Ciudad de México, (antes Distrito Federal) es la capital de la República Mexicana, se asienta sobre lo que fue el antiguo lago de Texcoco, cuna de la cultura Mexica y desde la época colonial se convirtió en la capital del Virreinato de la Nueva España. Ocupa una superficie de $1.485 \mathrm{~km}^{2}$ y se conforma por 16 Alcaldías (antes Delegaciones) (figura 1). En el 2015 concentró una población de 8,91 millones de habitantes (INEGI, 2015). El 28,4\% de su población vive con algún grado de pobreza, mientras el $1,7 \%$ de ésta vive en pobreza extrema (Estrategia de Resiliencia de la CDMX, 2016). Vale señalar que la CDMX aporta el $17,7 \%$ al PIB nacional y el sector terciario genera el $84,4 \%$ de su PIB interno, por otro lado, mientras que en 2010 captó el $60 \%$ de la inversión público-privada del país, para el 2012 captó el 65\% de la inversión extranjera a nivel nacional (SEDUVI, 2013).

Debido a la expansión urbana experimentada por la CDMX a lo largo de la segunda mitad del siglo $X X$, actualmente forma parte de la Zona Metropolitana del Valle de México (ZMVM) la cual se integra por las 16 Alcaldías de la CDMX, 59 municipios del Estado de México y 21 municipios del Estado de Hidalgo. Para el 2010, la ZMVM contaba con una población de 20,1 millones de habitantes, lo que representó el 17,9\% del total de la población nacional, que en ese momento rondaba los 112,3 millones de habitantes (INEGI, 2014). 
Para 2013, laboraban en la ZMVM 5,1 millones de personas en alguna actividad económica, lo que constituía el $23,6 \%$ del total nacional y generó para ese mismo año el $26,3 \%$ de la producción bruta nacional (INEGI, 2014). De esta manera, se estima que para el
2018, la ZMVM concentra una población de 21,8 millones de habitantes (CONAPO, 2012), lo que la convierte en la zona metropolitana más poblada de Latinoamérica.

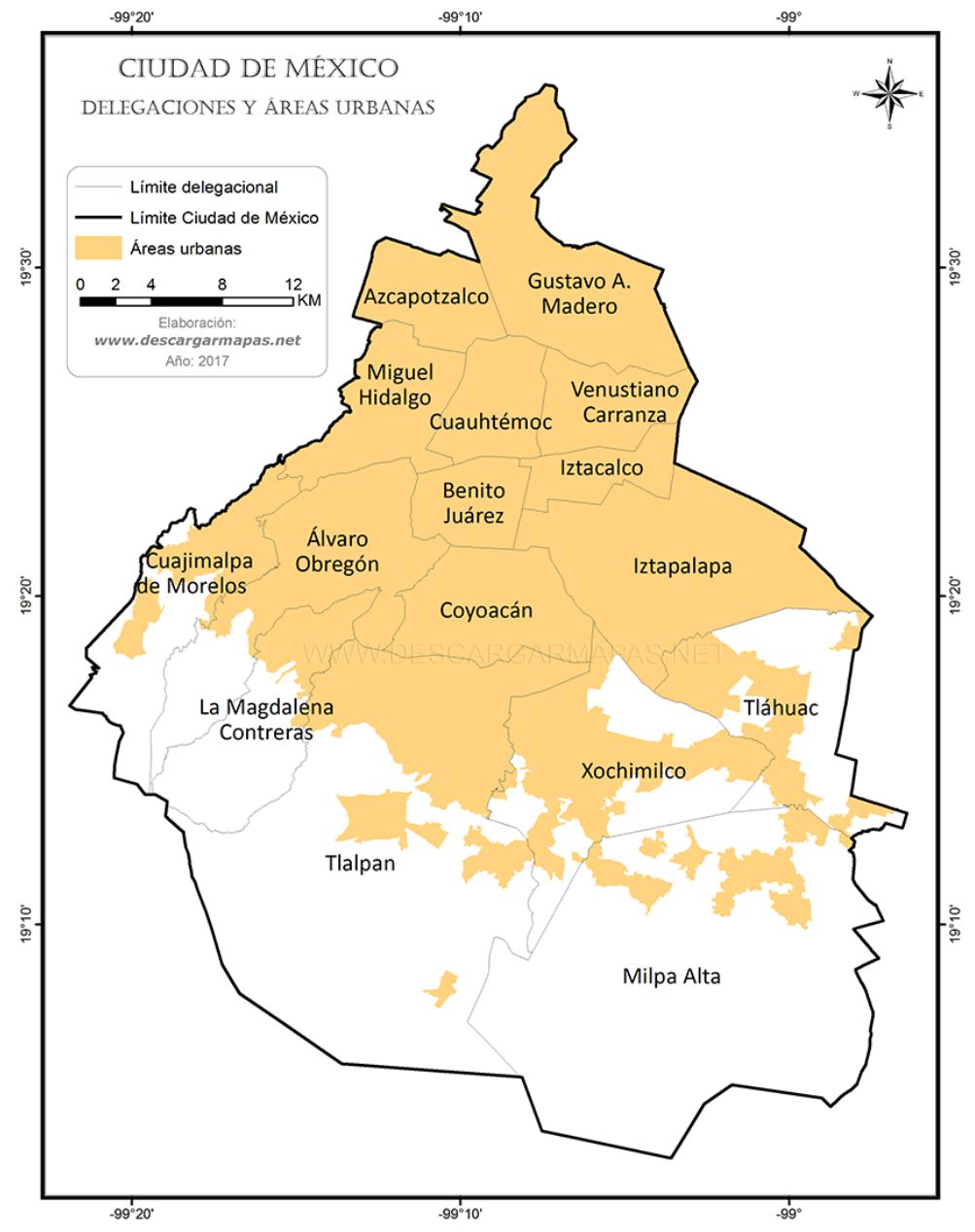

Figura 1. Alcaldías de la CDMX.

Fuente: https://descargarmapas.net/mexico/ciudad-de-mexico/mapa-ciudad-de-mexico-delegaciones-area-urbana

Para el análisis de nuestro caso de estudio encontramos desde este contexto, que tan sólo en la recién concluida administración (2012-2018) del gobierno capitalino, se avaló la edificación de 292 desarrollos inmobiliarios de alto impacto, que en conjunto suman $19.448 .912 \mathrm{~m}^{2}$ de construcción y de acuerdo a datos oficiales, los proyectos aprobados son en su gran mayoría desarrollos habitacionales, oficinas, plazas comerciales, hoteles y hospitales, algunos ya concluidos y otros en proceso de construcción. Las alcaldías que concentran la mayor cantidad de proyectos aprobados son: Álvaro Obregón con 46, que en conjunto suman 2.682.090 $\mathrm{m}^{2}$ de construcción; Miguel Hidalgo con 45, que en conjunto suman $3.291 .361 \mathrm{~m}^{2}$ de construcción; Benito Juárez con 36, que en conjunto suman un $1.827 .363 \mathrm{~m}^{2}$ de construcción y Cuauhtémoc con 32 , que en conjunto suman un $1.760 .333 \mathrm{~m}^{2}$ de construcción (tabla 1). Mientras que Cuajimalpa, es la delegación con el mayor número de metros cuadrados de construcción aprobados, con $3.327 .575 \mathrm{~m}^{2}$ en 31 desarrollos (Cruz-Flores, 2018) (figura 3). Los datos reflejan que tan sólo estas 5 alcaldías centrales 
concentraron el $66 \%$ del total de $\mathrm{m}^{2}$ de construcción avalados en toda la CDMX durante dicho período.

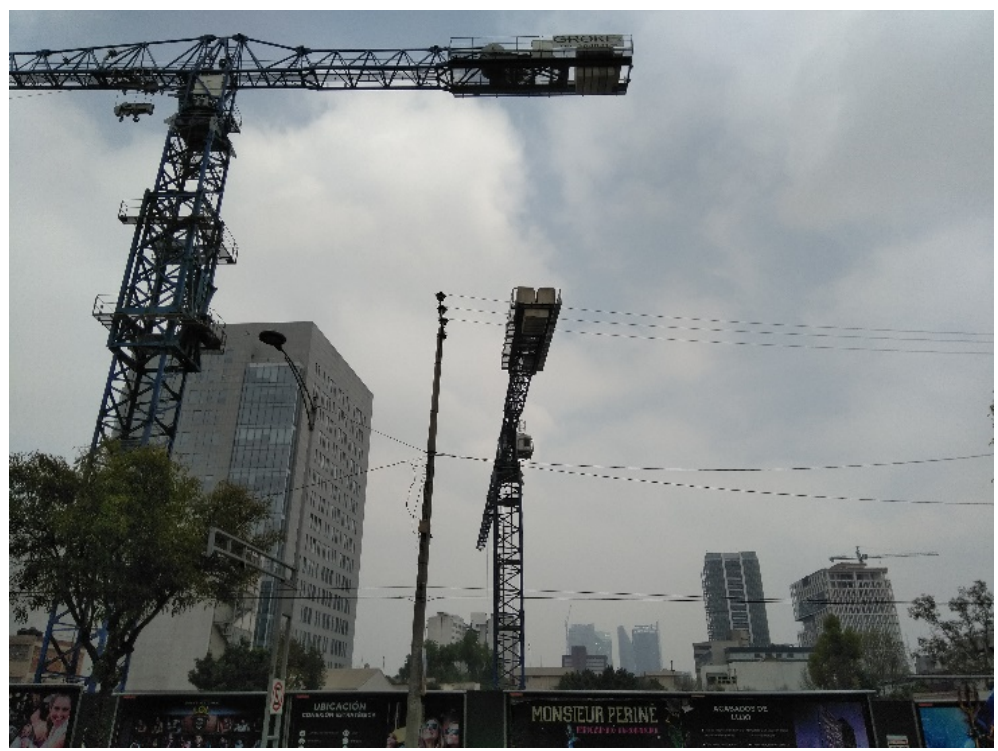

Figura 2. Desarrollos inmobiliarios en construcción en la Alcaldía Cuauhtémoc en diciembre de 2018.

Fuente: Colección personal.

Tabla 1

Desarrollos inmobiliarios de alto impacto avalados entre 2012 y 2018 en la CDMX

\begin{tabular}{ccc}
\hline Alcaldía & Número de desarrollos aprobados & Millones de $\mathbf{~ m}^{\mathbf{2}}$ de construcción \\
\hline Álvaro Obregón & 46 & 2.682 \\
Miguel Hidalgo & 45 & 3.291 \\
Benito Juárez & 36 & 1.827 \\
Cuauhtémoc & 32 & 1.760 \\
Cuajimalpa & 31 & 3.327 \\
Alcaldías restantes (11) & 102 & 6.561 \\
\hline Total CDMX & 292 & 19.448
\end{tabular}

Fuente: Elaboración propia con datos de Cruz-Flores, 2018.

La mayoría de las zonas donde se autorizaron estos proyectos experimentaron en tan sólo seis años un conjunto de rápidas y fuertes transformaciones urbanas que las pusieron en la mira del mercado inmobiliario principalmente por sus condiciones de centralidad, servicios, equipamiento, infraestructura o simplemente por localizarse en áreas consolidadas o bien conectadas de la ciudad, lo que en diversos casos generó no sólo su redensificación, sino la gentrificación de las mismas, lo que habla de la voracidad con la que el capital privado busca actualmente entrar en zonas de la ciudad consideradas hasta hace un par de años como "populares" lo que se traducía como áreas de baja rentabilidad o de poco atractivo económico para el mercado inmobiliario, aunque hoy en día la realidad es completamente diferente, porque el inminente $y$ excesivo crecimiento de la metrópoli hizo con el paso de los años, que adquirieran mayor valor de mercado, al 
quedar inmersas dentro de las áreas centrales consolidadas de la ciudad.

\section{EI descontento ciudadano ante la embestida del desarrollo inmobiliario}

Ante este escenario de crecimiento desbordado del mercado inmobiliario, los principales reclamos de los habitantes "oriundos" de estas zonas redensificadas giran en torno a que estos mega desarrollos inmobiliarios trastocan las características tradicionales de sus barrios, comunidades y colonias, al modificar de manera violenta su entorno urbano y los usos de suelo; dañan sus zonas patrimoniales; colapsan las vialidades y la infraestructura; generan grietas, hundimientos, socavones, congestión vial; privatizan el espacio público y aniquilan el muy mermado abastecimiento de agua potable, uno de los más graves problemas urbanos de la CDMX y del país.
Tan sólo en 2016, de acuerdo con datos del Instituto Nacional de Estadística y Geografía (INEGI), poco más de dos millones de hogares a nivel nacional carecían de agua potable, mientras que 4.341 .053 hogares la recibían cada tercer día y 1.305.304 hogares tan sólo la recibían una vez por semana, de un total de 32.920.270 de hogares a nivel nacional (INEGI, 2016) (tabla 2), lo que permite comprender el problema de abasto de agua potable en México, pero que en la CDMX es aún más grave, pues los datos indican que, mientras la disponibilidad promedio de agua por habitante en el país es de $3.982 \mathrm{~m}^{3} / \mathrm{hab} / \mathrm{año}$, en la CDMX es solamente de $152 \mathrm{~m}^{3} / \mathrm{hab} / \mathrm{año}$, cifra que denota el gran estrés hídrico al que está sujeta la cuenca del Valle de México (Estrategia de Resiliencia de la CDMX, 2016) y las altas complicaciones para dotar del vital líquido a esta ciudad ubicada a 2.250 msmn.

Tabla 2

Disponibilidad y frecuencia de agua en la vivienda por tamaño de localidad a nivel nacional, 2016

\begin{tabular}{|c|c|c|c|c|c|c|}
\hline \multirow{2}{*}{ Disponibilidad y frecuencia } & \multicolumn{2}{|c|}{ Total } & \multicolumn{2}{|c|}{$\begin{array}{c}\text { De menos de } 2.500 \\
\text { habitantes }\end{array}$} & \multicolumn{2}{|c|}{ De 2.500 y más habitantes } \\
\hline & Hogares & $\%$ & Hogares & $\%$ & Hogares & $\%$ \\
\hline Total & 32.925 .270 & 100 & 7.137 .808 & 100 & 25.787 .462 & 100 \\
\hline $\begin{array}{l}\text { Dentro de la vivienda o fuera de la } \\
\text { vivienda pero dentro del terreno }\end{array}$ & 30.840 .062 & 93,7 & 5.950 .327 & 83,4 & 24.889 .735 & 96,5 \\
\hline Diario & 22.428 .142 & 68,1 & 3.722 .779 & 52,2 & 18.705 .363 & 72,5 \\
\hline Cada tercer día & 4.341 .053 & 13,2 & 1.295 .405 & 18,1 & 3.045 .648 & 11,8 \\
\hline Dos veces por semana & 1.732 .243 & 5,3 & 390.469 & 5,5 & 1.341 .774 & 5,2 \\
\hline Una vez por semana & 1.305 .304 & 4,0 & 337.564 & 4,7 & 967.740 & 3,8 \\
\hline De vez en cuando & 1.033 .320 & 3,1 & 204.110 & 2,9 & 829.210 & 3,2 \\
\hline $\begin{array}{l}\text { Entubada de la llave pública, o que } \\
\text { acarrean de otra vivienda }\end{array}$ & 386.294 & 1,2 & 200.130 & 2,8 & 186.164 & 0,7 \\
\hline $\begin{array}{c}\text { De pipa, pozo, río, lago, arroyo u } \\
\text { otra }\end{array}$ & 1.698 .914 & 5,2 & 987.351 & 13,8 & 711.563 & 2,8 \\
\hline
\end{tabular}

Fuente: Encuesta Nacional de los Hogares, 2016. INEGI. http://www.beta.inegi.org.mx/contenidos/proyectos/enchogares/regulares/enh/2016/d oc/enh2016_resultados.pdf

De esta manera, el descontento ciudadano por la autorización, desarrollo y construcción de enormes complejos inmobiliarios se expresa a través de diversas acciones de protesta organizada, como son: manifestaciones, marchas, plantones, cierre de vialidades, enfrentamientos físicos, acciones legales, 
amparos y hasta la clausura simbólica de las obras en construcción. En todos los casos, los ciudadanos afectados dejan clara su postura: "NO! a la corrupción inmobiliaria" (González-Alvarado, 2018d) (figura 5); “el agua es un derecho humano", no un favor político" (González-Alvarado, 2018c); "No más condominios, queremos agua" (González-Alvarado, 2018b); "El daño ya está hecho, pero gracias a miles de vecinos logramos detener la voraz destrucción" (Sánchez-Jiménez, 2018);

Llevamos años pidiendo equipamiento, porque no hay ni mercado; tenemos que ir a las colonias vecinas. Pero eso sí, nos han llenado de edificios cuando el agua la recibimos por tandeo y no hay drenaje. En época de lluvias las inundaciones alcanzan hasta un metro y medio de altura (González-Alvarado, 2018a); "Masacre en Xoco por inmobiliarias voraces. En Nativitas, las megaconstrucciones nos han dejado sin agua" (González-Alvarado, 2018e).

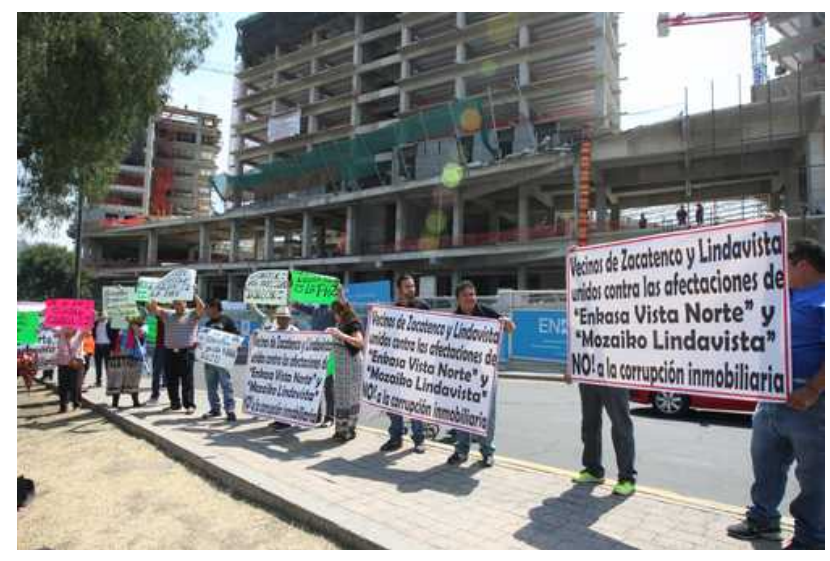

Figura 5. Habitantes de la delegación Gustavo A. Madero protestaron y clausuraron de manera simbólica dos desarrollos inmobiliarios que albergarán 550 viviendas, en la esquina de Acueducto y Cienfuegos. Foto Luis Humberto González.

Fuente: http://www.jornada.unam.mx/2018/03/02/capital/029n2cap

Otro ejemplo, son los desarrollos inmobiliarios emprendidos cerca de Ciudad Universitaria (CU), catalogada Patrimonio Cultural de la Humanidad por la UNESCO, este ejemplo resulta significativo por el hecho de que una de estas obras se ha detenido (clausurado) por acciones legales emprendidas por la propia Universidad Nacional Autónoma de México (UNAM), en contra del Gobierno de la CDMX, la Alcaldía de Coyoacán y la Secretaría de Desarrollo Urbano y Vivienda, alegando la violación de los tratados internacionales que protegen a las zonas declaradas patrimonio de la humanidad y que especifican zonas de amortiguamiento urbano para proteger dichos monumentos (Sánchez-Jiménez, 2018), pues de lo contrario, la UNESCO podría retirar la declaratoria de Patrimonio de la Humanidad al recinto universitario, considerado de los más icónicos e importantes complejos urbano-arquitectónicos del siglo $X X$.

Los casos citados tienen lugar en 9 de las 16 alcaldías: Gustavo A. Madero, Venustiano Carranza, Iztacalco, Cuauhtémoc, Benito Juárez, Coyoacán, Álvaro Obregón, Miguel Hidalgo y Azcapotzalco, lo que deja ver que las transformaciones se están dando en áreas urbanas consolidadas de las alcaldías centrales, destacando que varias de estas zonas eran consideradas años atrás como populares e incluso, fabriles, sin atractivo para el mercado inmobiliario porque en la mayoría de los casos la normatividad urbana se los impedía. Sin embargo, en los últimos seis años diversos grupos ciudadanos y organizaciones sociales han denunciado un fuerte $y$ sistemático incremento en la autorización ilegal -por cambiar de manera arbitraria el uso de suelo- de permisos para el desarrollo de grandes complejos inmobiliarios, caracterizados principalmente por 9 exclusivas torres de vivienda que oscilan entre los 6 y 30 pisos, que llegan a alcanzar los 840 condominios por conjunto (González y Quintero, 2018), además de que la mayoría cuenta con zonas de oficinas, centros comerciales y amplios estacionamientos.

La Comisión de Derechos Humanos de la CDMX (CDH) ha realizado un informe a partir del análisis de 234 quejas relacionadas con temas de desarrollo urbano, (150 quejas individuales y 84 colectivas - una promovida por residentes de más de 700 colonias de las 16 demarcaciones territoriales-) en las que se documenta la continua violación a diversas normas jurídicas en 1.200 obras por: cambios de uso de suelo, inobservancia de los programas delegacionales y parciales de desarrollo urbano, falta de armonización legislativa para evitar la discrecionalidad en el otorgamiento de permisos, licencias, manifestaciones de construcción y respecto de los criterios de redensificación de predios (BolañosSánchez, 2018), razón por la cual, diputados de la actual legislatura de la Ciudad de México, realizaron la propuesta para que la $\mathrm{CDH}$ pueda dictar medidas de observancia obligatoria cuando un ciudadano denuncie la 
violación de sus derechos por alguna construcción (figuras 6 y 7), además de establecer en la ley sanciones más severas tantos para desarrolladores como para servidores públicos (Bolaños-Sánchez, 2018), para dar cumplimiento al mandato de la Constitución Política de la Ciudad de México, algo sin precedentes a nivel nacional, al ser la CDMX, la primera en reconocer de manera legal el Derecho a la Ciudad como un derecho humano fundamental de todos sus habitantes (Instituto de Investigaciones Parlamentarias, 2017).

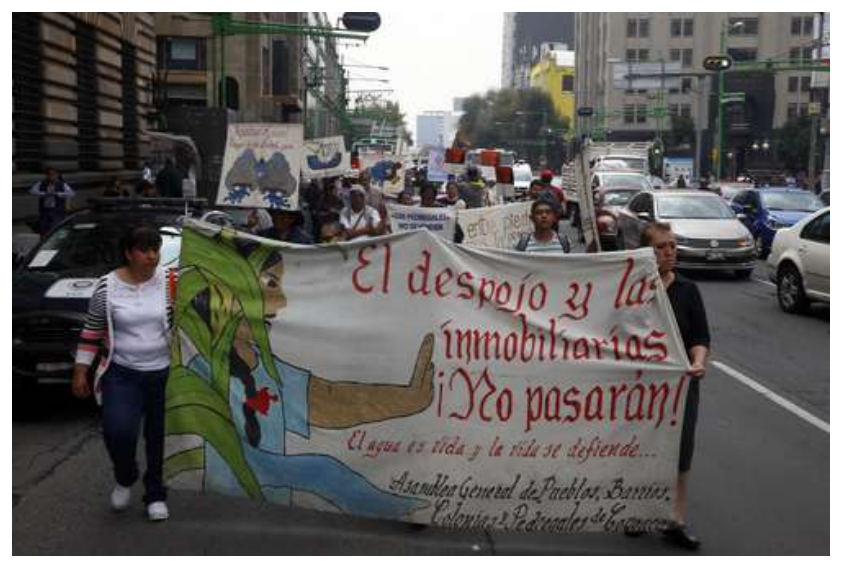

Figura 6. Organizaciones vecinales marcharon del Hemiciclo a Juárez al Congreso local en protesta por la corrupción con que se han autorizado megaconstrucciones. Foto Jesús Villaseca.

Fuente: https://www.jornada.com.mx/2018/10/12/capital/036n3cap\#

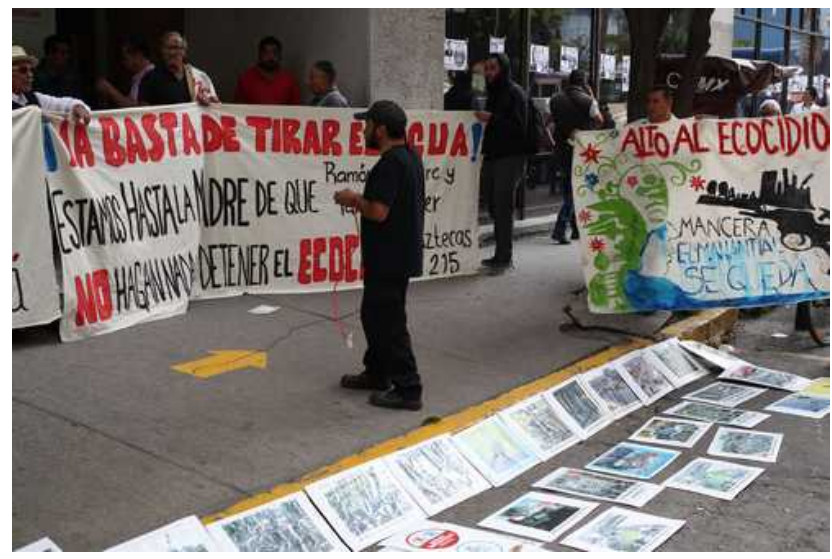

Figura 7. La Asamblea General de los Pueblos, Barrios, Colonias y de los Pedregales de Coyoacán realizaron un mitin en defensa del agua afuera de las oficinas del Sistema de Aguas de la Ciudad de México. Foto José Antonio López.

Fuente: http://www.jornada.unam.mx/2018/03/22/capital/034n2cap

Los casos anteriores permiten entender la magnitud del problema generado por la voracidad del mercado inmobiliario en los últimos 6 años de gobierno local y que la mayoría de los casos, bajo el argumento de la densificación de zonas urbanas consolidadas, emprenden la construcción de gigantescos complejos inmobiliarios que lejos de cumplir el objetivo de la densificación, definida por la Ley Federal de Asentamientos Humanos Ordenamiento Territorial y Desarrollo Urbano (LGAH) (2016) como la acción de "incrementar el número de habitantes y la población flotante por unidad de superficie, considerando la capacidad de soporte del territorio y, en su caso, adecuando los espacios públicos y sus infraestructuras" (p. 3).

Estos macro desarrollos inmobiliarios buscaron tan sólo obtener el mayor beneficio económico de la inversión, sin atender, que todos los habitantes de la ciudad "tienen derecho a vivir y disfrutar Asentamientos Humanos sustentables, resilientes, saludables, productivos, equitativos, justos, incluyentes, democráticos y seguros" (LGAH, p. 2), como ocurre con el Sistema de Actuación por Cooperación de la zona denominada Granadas, mejor conocida como Nuevo Polanco, integrada por 12 colonias de la Alcaldía Miguel Hidalgo, de la cual, la administración local anterior aseguró "el mejoramiento y consolidación sustentable del desarrollo urbano, a través del mejoramiento de la zona mediante la promoción y apoyo de infraestructura y equipamiento, y el desarrollo de servicios públicos, vivienda, comercio, recreación y turismo" (SEDUVI, 2015).

Sin embargo, el Comité ciudadano de vigilancia de Parques Polanco, expone que lo que en realidad ocurre en el Polígono Granadas es ejemplo de cuando no existe planeación urbana:

El impacto es brutal; se nos juntan todos los problemas, que padecemos los que ya vivían aquí y los que llegamos. No hay abasto suficiente de agua, el drenaje está colapsado, es tortuoso circular por las calles y no hay espacios de recreación (González y Quintero, 2018).

Argumentan además que:

no hay obras de mitigación de las inmobiliarias, ni reforzamiento de la infraestructura por las autoridades, lo que llevó a que el año pasado, durante los torrenciales aguaceros, los sótanos de los nuevos edificios de Las Granadas se inundaran (González y Quintero, 2018). 
Este caso ejemplifica de manera puntual los efectos perversos de las transformaciones urbanas orientadas en exclusiva al mayor beneficio económico del mercado inmobiliario, a través de una supuesta redensificación que desborda los límites permisibles de estas zonas, lo que sin lugar a duda representa una clara violación del artículo 12 (Derecho a la Ciudad) de la Constitución Política de la Ciudad de México.

\section{Conclusiones}

Al hacer un análisis de éstos y otros tantos casos similares documentados por la prensa, de la situación que hoy se vive en 9 de las 16 alcaldías de la CDMX, se afirma junto con Harvey (2008), que la urbanización capitalista es una mera actividad rentable y que la urbanización ha jugado un papel crucial en la absorción de excedentes de capital, a escalas geográficas cada vez mayores, pero el precio de los procesos florecientes de "destrucción creativa" han desposeído a las masas de cualquier Derecho a la Ciudad en absoluto (p. 37).

Los cambios experimentados por las ciudades y la realidad urbana por lo menos a lo largo del último siglo, a través del urbanismo fordo-keynesiano-corbusiano propio de la segunda modernidad (Ascher, 2004) impactaron de manera definitiva no sólo la configuración formal-funcional de éstas, sino la manera en la que los usuarios y habitantes de la ciudad la identifican, viven y significan. Por esta razón, en pleno siglo XXI y con una tercera modernidad a toda marcha (Ascher, 2004), cobra particular importancia el espacio urbano en la conformación de la identidad, pues cuando el habitante establece un vínculo emocional o afectivo con aquellos espacios que le son cercanos o propios, éstos fundamentan su vida, sus recuerdos y su memoria, lo que se hace extensivo a todos los integrantes de la sociedad, dando a la vez, identidad a los propios espacios.

De esta manera, surge un cuestionamiento final ¿Qué relación tienen entre sí Derecho a la Ciudad, identidad urbana, apropiación simbólica del espacio y la defensa social del espacio urbano? La respuesta es contundente: cuando los individuos generan identidad con los espacios de la ciudad que en su cotidianidad habitan, se produce la apropiación simbólica de éstos, proceso que a lo largo del tiempo genera vínculos afectivos con el entorno, lo que da paso a la acción colectiva organizada en defensa del territorio, los recursos y el espacio urbano, por encima del simple usufructo e intereses económicos del mercado inmobiliario, lo que genera que el espacio urbano adquiera su verdadera connotación como un espacio simbólico socialmente edificado.

En esencia, esto es lo que se puede concluir de los diferentes movimientos sociales analizados y que luchan por la defensa del espacio urbano, no por una simple oposición al proceso de urbanización del que forman parte, sino por el hecho de que los cambios experimentados en los últimos años (redensificación y regeneración urbana de zonas centrales en la CDMX) representan violentas transformaciones del entorno que quebrantan sus recursos (suelo y agua), colapsan su infraestructura y devastan su espacio público, además de que alteran la conformación socio-espacial de las zonas intervenidas al gentrificarlas $y$ atentan contra la memoria, imaginario e identidad de los habitantes, lo que constituye una clara violación al artículo 12 (Derecho de la Ciudad) de la Constitución Política de la Ciudad de México.

Por tal motivo, la identidad urbana se erige como un elemento fundamental para el análisis de la realidad socio-espacial actual. Por su parte, el estudio del espacio urbano y la apropiación simbólica que de él hacen sus usuarios, permite comprender la manera en la que los individuos y las colectividades hacen ciudad de la mano de diversos procesos, aptitudes y competencias como es el caso de la urbanidad, la civilidad y la ciudadanía, que en conjunto implican el desarrollo de la sociabilidad urbana (Giglia, 2012), además de posibilitar el análisis de las problemáticas que actualmente enfrentan las ciudades contemporáneas.

Los actuales movimientos ciudadanos que de manera global resurgen en oposición al voraz mercado inmobiliario (como se dio en las décadas de los 70 y 80 del siglo pasado, posterior a la formulación teórica del derecho a la ciudad por Lefebvre) y que se ejemplifican a través de los casos analizados de la CDMX, representan un conjunto de acciones ciudadanas organizadas que de manera libre, consciente y colectiva pugnan por un uso sustentable, justo e incluyente del espacio urbano en beneficio de sus habitantes, lo que se traduce en el respeto y cumplimiento constitucional del Derecho a la Ciudad, lo que marca el inicio de una nueva etapa, en donde la participación activa de la ciudadanía podrá 
incidir en el rumbo que tomarán las políticas públicas que se apliquen al futuro desarrollo urbano de la ciudad por parte de las administraciones locales en turno, lo que representa una condición de avanzada a nivel latinoamericano.

Es por ello que en los ejemplos seleccionados queda de manifiesto que cuando estos procesos identitarios y de apropiación simbólica del espacio se generan, la movilización ciudadana surge para defender el entorno y sus recursos de las transformaciones que de manera arbitraria, violenta e ilegal alteran su realidad socio espacial porque, al habitar estos espacios por generaciones, sus habitantes se reconocen como parte de un grupo, con raíces y una historia común con el territorio, que se materializa en el espacio urbano que habitan y que forma parte de sus recuerdos, historia y memoria colectiva.

Lo anterior se convierte en un argumento a favor de un quehacer urbano-arquitectónico cuyas acciones se desarrollen en un marco ético, que garantice a todos sus habitantes el acceso a condiciones dignas de vida, con un medio ambiente limpio, digno y saludable; donde el
Patrimonio Cultural de la ciudad (arquitectónico, artístico y de monumentos) sea respetado, valorado y conservado como parte fundamental de la Cultura e identidad de todos sus habitantes y en donde la ciudad garantice un futuro planeado y sostenible para las futuras generaciones, como parte de esa ciudad para todos, fruto de correctas políticas públicas y de una práctica urbano-arquitectónica sustentable, resiliente y responsable socialmente, que redensifique las zonas urbanas consolidadas que así lo permitan, más allá de la maximización del lucro económico, la especulación inmobiliaria y la gentrificación de las mismas a costa del bien de las clases más desfavorecidas.

De esta manera se puede avanzar hacia la concreción del Derecho a la Ciudad, lo que finalmente permitirá la construcción de ciudades más sustentables, resilientes, justas y equitativas para todos sus habitantes, garantizando así el futuro de las próximas generaciones, pues como bien afirma Harvey (2008), "el derecho a la ciudad es mucho más que la libertad individual para acceder a los recursos urbanos: es un derecho a cambiar nosotros mismos cambiando la ciudad" (p. 23) [B] 


\section{Referencias}

Aguilar, M.A. (2012), Antropología urbana y lugar. Recorridos conceptuales, en Angela Giglia, y Amalia Signorelli (coords.), Nuevas topografías de la cultura, México: UAM-Iztapalapa-Juan Pablos Editor.

Alva, B. y Aldrete, L. (2011). Identidad Urbana en San Luís Potosí a través de la Percepción social en el año 2011, en Ruiz, H. (et. al.), (coords.). Diversidad Cultural, Identidades y Territorio: adscripción, apropiación y recreación. Recuperado de: http://www.eumed.ne t/libros/2012a/1149/indice.htm

Ascher, F. (2004). Los nuevos principios del urbanismo. El fin de las ciudades no está a la orden del día. Madrid: Alianza Editorial.

Bauman, Z. (2007). Los Retos de la educación en la Modernidad Líquida, Argentina: Gedisa.

Bolaños-Sánchez, A. (05 de diciembre 2018). Diputados plantean dar más facultades a PAOT y CDH contra inmobiliarias. La Jornada. Recuperado 05 de diciembre de 2018 de https://www.jornada.com .mx/2018/12/05/capital/039n1cap\#

Carta Mundial por el Derecho a la Ciudad. (2013). Cuadernos Geográficos, 52. Recuperado de http://www.redalyc.org/articulo.oa?id=17128112016

CONAPO (2012). Proyecciones de población de zonas metropolitanas de México 2010-2030. Recuperado de https://bit.ly/2Q8vwdf

Constitución Política de la Ciudad de México (2017). Recuperada de: http://infodf.org.mx/documentospdf/constitucion cd mx/Constitucion \%20Politica CDMX.pdf

Contreras, Y. (2017). De los "gentries" a los precarios urbanos. Los nuevos residentes del centro de Santiago. EURE, 43(129), 115-141. https://doi.org/10.4067/s0250-71612017000200006

Corona-Rodríguez, J. (2013). Etnografía de lo virtual: experiencias y aprendizajes de una propuesta metodológica para investigar internet. Razón y Palabra, 18(83). Recuperado de https://www.reda lyc.org/articulo.oa?id=199527531045
Cruz-Flores, A. (03 de diciembre 2018). Invadieron la ciudad 19.4 millones de $\mathrm{m}^{2}$ de concreto y acero en esta administración. La Jornada. Recuperado 06 de diciembre de 2018 de https://www.jornada.co m.mx/2018/12/03/capital/026n1cap\#

Flores-Vivar, J. (2018). Ciberantropología del periodismo en red. Enfoques teóricos y propuestas metodológicas para el estudio de la audiencia digital. Letras, 89(129), 4-29. http://dx.doi.org/10.30920/le tras.89.129.1

Giglia, A. (2012). El Habitar y la Cultura. Perspectivas Teóricas de Investigación. España: UAM-IztapalapaAnthropos.

Gómez-Carmona, G. y Villar-Calvo, A. (2015). Impactos de lo global en lo local: Gentrificación en ciudades latinoamericanas. Revista de Urbanismo, 32, 3-17. https://doi.org/10.5354/0717-5051.2015.36553

Gómez-Carmona, G., Villar-Calvo, A. e Inzulza-Contardo, J. (2016). La reconfiguración urbana de ciudades intermedias mexicanas en el contexto latinoamericano. El caso de Metepec, México. AUS, 19, 66-72. https://doi.org/10.4206/aus.2016.n19-11

González-Alvarado, R. (14 de febrero 2018a). Aunado al olvido oficial Santa Úrsula Coapa enfrenta el asedio inmobiliario. La Jornada. Recuperado 11 de marzo 2018 de http://www.jornada.unam.mx/2018/02 L14/capital/031n1cap

González-Alvarado, R. (20 de febrero 2018b). Lunes de protestas y caos vial por la falta de agua en cuatro delegaciones. La Jornada. Recuperado 18 de marzo 2018 de http://www.jornada.unam.mx/2018/02 /20/capital/029n1cap

González-Alvarado, R. (27 de febrero 2018c). Vecinos de Los Pedregales exigen a Toledo no utilizar el agua con fines partidistas. La Jornada. Recuperado 18 de marzo 2018 de http://www.jornada.unam.mx/20 18/02/27/capital/032n3cap

González-Alvarado, R. (02 de marzo 2018d). Clausuran colonos de GAM 2 desarrollos inmobiliarios. $L a$ Jornada. Recuperado 02 de marzo de 2018 de http://www.jornada.unam.mx/2018/03/02/capital/0 29n2cap 
González-Alvarado, R. (12 de octubre 2018e). Capitalinos exigen un ya basta al caos inmobiliario. La Jornada. Recuperado 06 de diciembre de 2018 de https://www.jornada.com.mx/2018/10/12/capital/03 6n3cap\#

González, R. y Quintero, J. (04 de diciembre 2018). La oleada inmobiliaria en este sexenio impactó servicios y la vida de capitalinos. La Jornada. Recuperado 04 de diciembre de 2018 de https://www.jornada.c om.mx/2018/12/04/capital/032n1cap\#

Harvey, D. (2008). The Right to the City. New Left Review, 53, 23-40. Recuperado de https://newleftreview.o $\mathrm{rg} / \mathrm{Il} / 53 /$ david-harvey-the-right-to-the-city

INEGI (2014). Minimonografía las Zonas Metropolitanas en México. Censos económicos. Recuperado de https://bit.ly/2rYIOuh

INEGI (2015). Información de México para niños. Recuperado de https://bit.ly/1D7e4tp

INEGI (2016). Encuesta Nacional de los Hogares 2016. Recuperado de https://bit.ly/2whylmm

Instituto de Investigaciones Parlamentarias (2017). El Derecho a la Ciudad en la Constitución de la Ciudad de México. Una propuesta de interpretación. Recuperado de https://bit.ly/2AIFVxc

Inzulza-Contardo J. y Galleguillos, X. (2014). Latino gentrificación y polarización: transformaciones socioespaciales en barrios pericentrales y periféricos de Santiago, Chile. Revista de Geografía Norte Grande, 58, 135-159. https://doi.org/10.4067/s071834022014000200008

Inzulza-Contardo, J. y Díaz, I. (2016). Desastres naturales, destrucción creativa y gentrificación: estudio de casos comparados en Sevilla (España), Ciudad de México (México) y Talca (Chile). Revista de Geografía Norte Grande, 64, 110-128. https://doi.org/10.4067/s071834022016000200008

Janoschka, M., Sequera, J. \& Salinas L. (2013). Gentrification in Spain, and Latin America, - a Critical Dialogue. International Journal of Urban and Regional Research. 38(4), 1234-1265. https://doi.org/10.1111/1468-2427.12030

LGAH - Ley General de Asentamientos Humanos, Ordenamiento Territorial y Desarrollo Urbano. (2016).
Recuperado de http://www.diputados.gob $. \mathrm{mx} /$ LeyesBiblio/pdf/LGAHOTDU 281116.pdf

Magrini, Ma. y Cataláo, I. (2017). Del derecho al consumo, al derecho a la ciudad: contradicciones y convergencias. Eure, 43(130), 25-46. https://doi.org/10.4067/s0250-71612017000300025

Montaner, J. y Muxí, Z. (2011), Arquitectura y Política. Barcelona: Gustavo Gili.

Nueva Agenda Urbana (2017). Recuperado de http://habitat3.org/wp-content/uploads/NUASpanish.pdf

Sánchez-Jiménez, A. (08 de marzo 2018). Construcciones a las afueras del campus lastiman la arquitectura patrimonio universal de la UNESCO. La Jornada. Recuperado 08 de marzo de 2018 de https://www.jornada.unam.mx/2018/03/08 Lsociedad/037n2soc

SEDUVI (2013). Agenda hacia una ciudad compacta, dinámica, policéntrica y equitativa. Recuperado de https://bit.ly/2Q8dVlv

SEDUVI (2015). Coordinará SEDUVI acciones, obras y proyectos estratégicos para mejoramiento $y$ consolidación en regeneración de la zona granadas. Recuperado de https://bit.ly/2Rko03v

Tamayo, S. y Wildner K. (coords.) (2005). Identidades urbanas, México: UAM.

Valera, S. (1996). Análisis de los aspectos simbólicos del espacio urbano. Perspectivas desde la psicología ambiental. Revista de Psicología Universitas Tarraconensis, 18(1), 63-84. Recuperado de https://bit.ly/2Q87krB

Vidal, T. y Pol E. (2005). La apropiación del espacio: una propuesta teórica para comprender la vinculación entre las personas y los lugares. Anuario de Psicología, 36(3), 281-297. Recuperado de http://www.redalyc.org/articulo.oa?idp=1\&id=97017 $406003 \&$ cid $=4374$

Waldmann, A. (2011). Estilos de Consumo y conformación de Identidades en santa Cruz de la Sierra. En Guaygua, G. (et. al.). Nuevas Identidades Urbanas, Tres Miradas desde la Cultura y la Desigualdad. Recuperado de https://bit.ly/2RpD1kA 